\title{
First confirmed record of the ant genus Myrmecina (Hymenoptera, Formicidae) from the Malay Peninsula: description of a new species and a key to Myrmecina species from Sundaland
}

\author{
Mark K. L. Wong', Benoit Guénard ${ }^{2}$ \\ I National Parks Board, Singapore Botanic Gardens, 1 Cluny Road, Singapore 2 School of Biological Sciences, \\ The University of Hong Kong, Pokfulam Road, Hong Kong \\ Corresponding author: Benoit Guénard (bguenard@hku.hk) \\ Academic editor: Michael Obl | Received 29 March 2016 | Accepted 20 April 2016 | Published 27 June 2016 \\ http://zoobank.org/8B72E213-BFDC-4334-AD6E-C2ED1067C231 \\ Citation: Wong MKL, Guénard B (2016) First confirmed record of the ant genus Myrmecina (Hymenoptera, Formicidae) \\ from the Malay Peninsula: description of a new species and a key to Myrmecina species from Sundaland. Journal of \\ Hymenoptera Research 50: 129-140. doi: 10.3897/JHR.50.8652
}

\begin{abstract}
We present the first confirmed record of the little known and uncommon ant genus Myrmecina for the Malay Peninsula. Myrmecina magnificens sp. n., a new species displaying unique anteriorly pointing propodeal spines, is described from specimens of the worker caste collected in a selectively logged primary rainforest in Singapore. We also provide the first key to Myrmecina species of the Sundaland region.
\end{abstract}

\section{Keywords}

Myrmecina, Southeast Asia, Malay Peninsula, Singapore, Leaf litter 


\section{Introduction}

Ants of the myrmicine genus Myrmecina are generally encountered in leaf litter samples where they live in small colonies of 30 to less than 150 individuals (Buschinger and Schreiber 2002; Terayama et al. 2014). Little is known about their biology, although specialized predation on oribatid mites has been observed in two Japanese species (Masuko 1994). Some Myrmecina species are also known to exhibit queen polymorphism, where two different phenotypes of reproductive females occur within a colony (Steiner et al. 2006). The genus contains 51 valid species (excluding the current description) and 1 valid subspecies (Bolton 2016). These ants are distributed throughout the Nearctic, Palearctic, Indomalayan and Austral regions, and apparently absent from Central and South America, sub-Saharan Africa and most of the Middle East (Antmaps 2016). Within the Indomalayan region, Myrmecina has been recorded from India and Sri Lanka (4 species), China and Taiwan (10 species), Myanmar (1 species) as well as the Sundaland (6 species) (Antmaps 2016). Most of the recent taxonomic work on Myrmecina has focused on Chinese species (Huang et al. 2008, Zhou et al. 2008), while other Oriental members of the genus from tropical regions were described nearly a century ago, in particular species from the Sundaland region. Here, we describe a new Myrmecina species possessing distinct anteriorly pointing propodeal spines, which was discovered in a selectively logged primary forest of Singapore. The discovery of Myrmecina magnificens sp. $\mathrm{n}$. represents the first confirmed record of the Myrmecina genus from the Malay Peninsula. We also provide a key for Myrmecina species from Sundaland and highlight the need for more extensive sampling of ant communities in this part of the world.

\section{Methods}

Pictures of specimens were obtained with an incorporated digital camera, Leica DFC450, mounted on a Leica M205C dissecting microscope through the Leica Application Suite V4 software. A total of 24 to 86 images were taken and stacked together. Measurements of specimens were taken in $\mathrm{mm}$ (accurate to $0.001 \mathrm{~mm}$ and rounded to the nearest $0.01 \mathrm{~mm}$ for presentation) with the Measure Tools function of the Leica Application Suite V4 software on imaged specimens after proper placement for each body part measured. Measurements and morphological terminology follow: Shattuck (2009) for HW, HL, SL and WL; Fischer et al. (2014) for PNH and PTH; Baroni Urbani (1977) for PI and PPI.

The abbreviations used for the measurements and indices are as follows:

HW Head Width. Maximum width of head in full-face view excluding the eyes. HL Head Length. Maximum head length in full-face view, measured from the anterior-most point of the clypeal margin to the midpoint of a line drawn across the posterior margin of the head. 
MaL Mandible Length. Maximum length of mandible from the anterolateral margin of clypeus at outer side of mandibular insertion to mandibular apex.

SL Scape Length. Scape length, excluding the basal radicle.

EL Eye Length. Maximum diameter of eye measured in lateral view.

TL Total Length. Maximum length of specimen measured from the tip of the mandibles to the tip of the abdominal segment VII, not including sting. Due to the position of the specimen, total length was measured as the sum of head length + thorax, petiole and postpetiole length + gaster length.

WL Weber's length measured from the anterior-most point of the pronotal collar to the posterior-most point of the propodeal process.

PNH Pronotum Height. Maximum height of pronotum, measured in profile from the posterior base of the lateral sides of pronotum, where procoxa is attached, to the highest point of the pronotum.

PNW Pronotal Width. Maximum width of pronotum measured in dorsal view.

MW Mesonotal Width. Maximum width of the mesonotum measured in dorsal view.

PSL Propodeal Spine Length. Maximum length of propodeal spines measured in profile view from the tip of the propodeal spine to the closer outward margin of the propodeal spiracle.

PTL Petiole Length. Maximum diagonal length of petiole, measured in lateral view, from most anteroventral point of the peduncle, at or below the propodeal lobe, to most posterodorsal point at the junction with helcial tergite.

PTH Petiole Height. Maximum height of petiole, measured in lateral view from the highest (median) point of the node, orthogonally to the ventral outline of the node.

PTW Petiole Width. Maximum width of the petiole in dorsal view.

PPL Postpetiole Length. Maximum length of postpetiole, measured in lateral view.

PPH Postpetiole Height. Maximum height of postpetiole, measured in lateral view from the highest point of the node.

PPW Postpetiole Width. Maximum width of the postpetiole in dorsal view.

CI Cephalic Index. Calculated as: HW / HL $\times 100$.

SI Scape Index. Calculated as: SL $/ \mathrm{HW} \times 100$.

MaI Mandibular Index. Calculated as: $\mathrm{MaL} / \mathrm{HW} \times 100$.

PI Petiolar Index. Calculated as: PTW / PTL $\times 100$.

PPI Postpetiolar Index. Calculated as: PPW / PPL $\times 100$.

Abbreviations of the type depositories and others are as follows:

LKCNHM Lee Kong Chian Natural History Museum, Singapore.

SBSHKU Insect Biodiversity and Biogeography Laboratory, School of Biological Sciences, The University of Hong Kong, Hong Kong SAR. 


\section{Results}

\section{Description of new species}

\section{Myrmecina magnificens Wong \& Guénard, sp. n.}

http://zoobank.org/DA28E247-B301-4642-8250-729C0F05AC66

Figs $1-5$

Holotype. Worker from SINGAPORE, Seletar Trail, Central Catchment Nature Reserve, $1.395141^{\circ} \mathrm{N}, 103.802595^{\circ} \mathrm{E}, 47 \mathrm{~m}, 02 . \mathrm{IX} .2015$, leaf litter, leg. Mark K. L. Wong, label "MW020915-L1.1" (ANTWEB1009004) deposited in LKCNHM.

Paratypes. Four workers in total (ANTWEB1009005, ANTWEB1009006, ANTWEB1009007, ANTWEB1009008), all with the same collection data as holotype (deposited at SBSHKU).

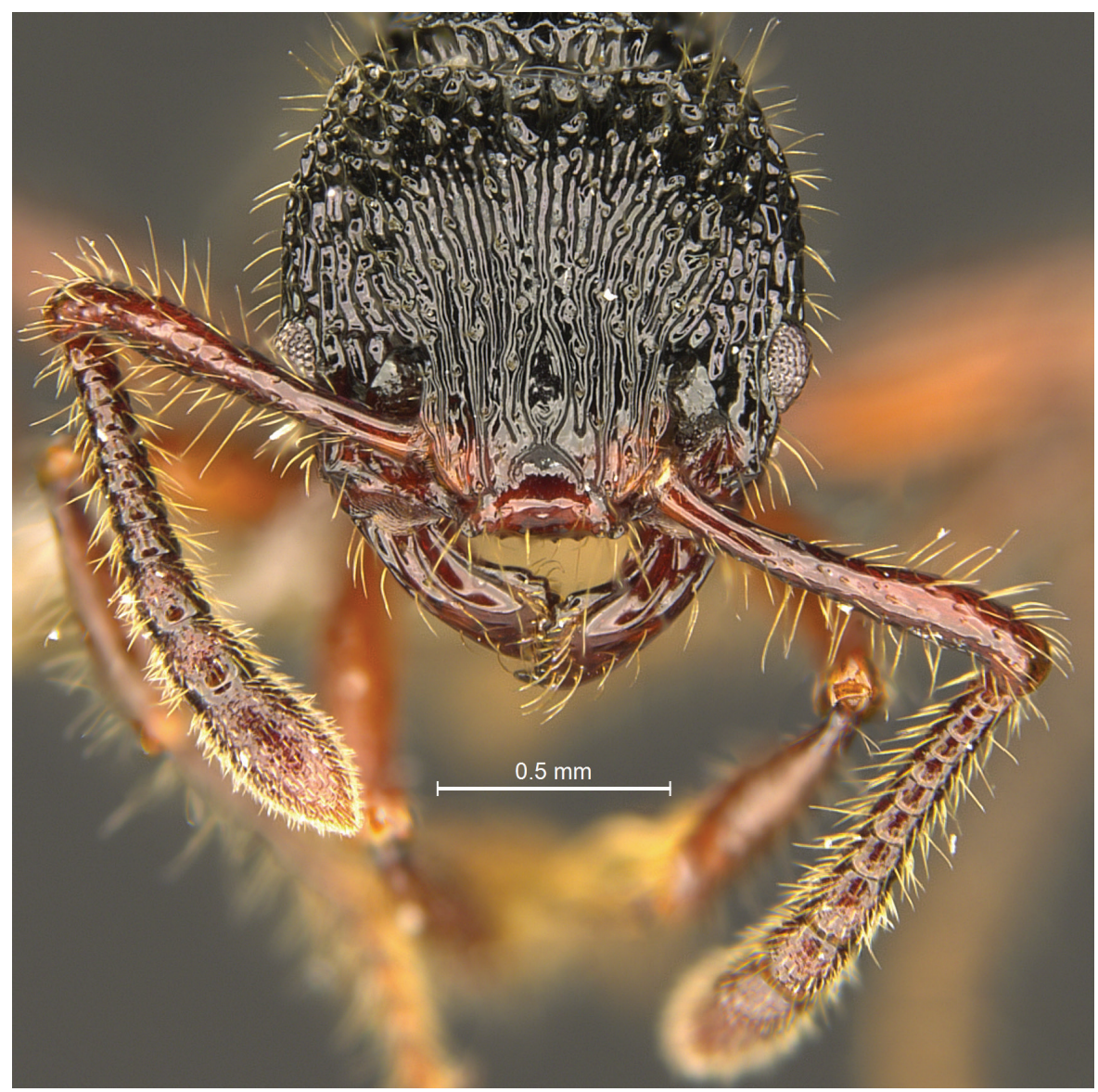

Figure I. Full-face view of Myrmecina magnificens (Holotype, ANTWEB1009004, LKCNHM) from Singapore. 


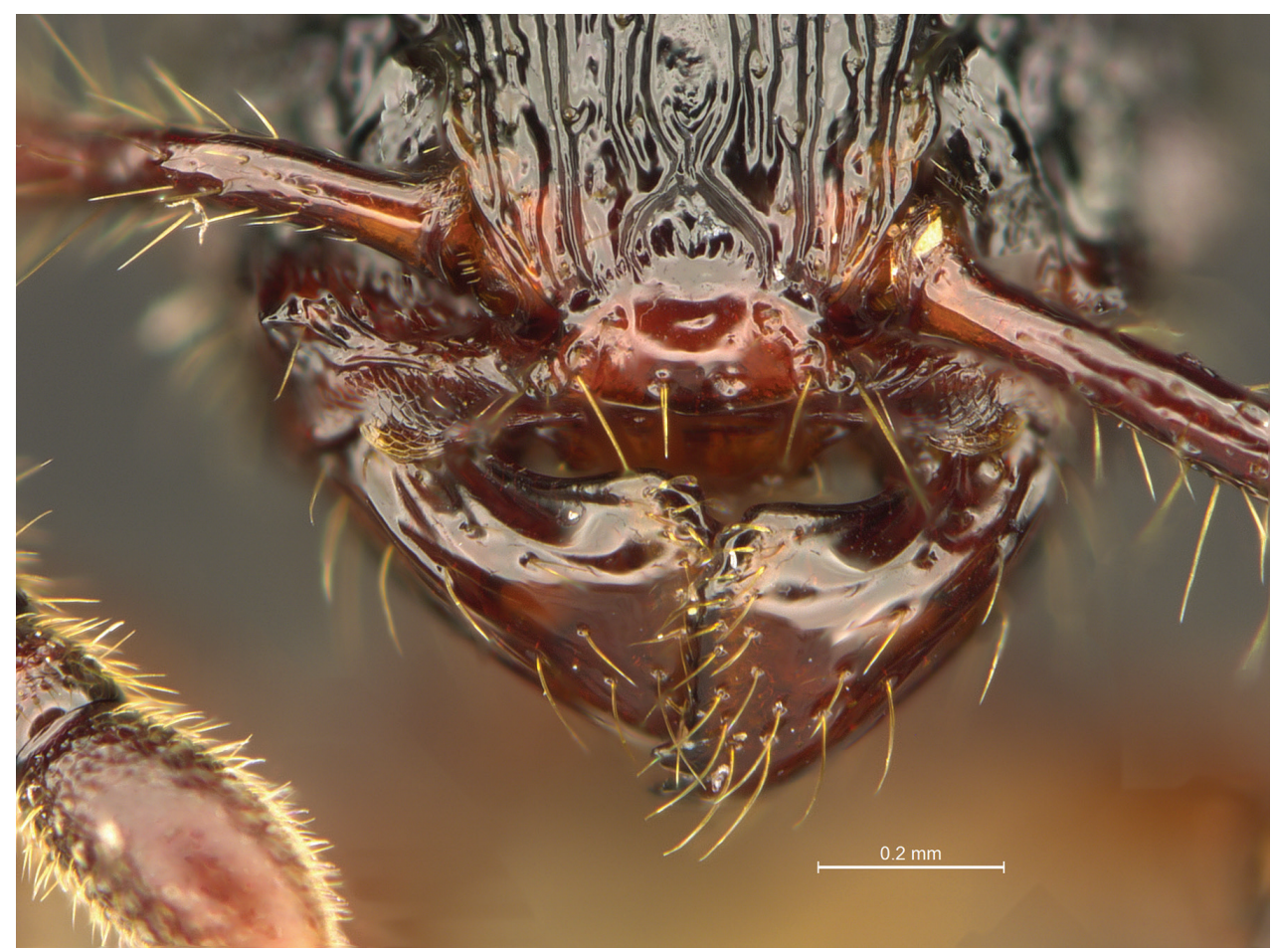

Figure 2. Head view focusing on mandibles of Myrmecina magnificens (Holotype, ANTWEB1009004, LKCNHM) from Singapore.

Measurements and indices. Holotype: HL $0.98 \mathrm{~mm}$; HW $1.12 \mathrm{~mm}$; MaL 0.63 mm; SL 0.90 mm; EL 0.20 mm; WL 1.37 mm; PNW $0.71 \mathrm{~mm}$; PNH $0.62 \mathrm{~mm}$; MW $0.65 \mathrm{~mm}$; PSL $0.24 \mathrm{~mm}$; PTL $0.36 \mathrm{~mm}$; PTW $0.32 \mathrm{~mm}$; PTH $0.35 \mathrm{~mm}$; TL 4.42 mm (stinger not included); PPL $0.23 \mathrm{~mm}$; PPW $0.34 \mathrm{~mm}$; PPH $0.39 \mathrm{~mm}$; CI 114, SI 80, MaI 56, PI 88, PPI 149.

Paratypes (n=4): HL 0.94-0.98 mm; HW 1.06-1.11 mm; MaL 0.55-0.63 mm; SL 0.89-0.91 mm; EL 0.20-0.21 mm; WL 1.22-1.36 mm; PNW 0.70-0.74 mm; PNH 0.57-0.62 mm; MW 0.61-0.65 mm; PSL 0.23-024 mm; PTL 0.34-0.36 mm; PTW 0.29-0.31 mm; PTH 0.31-0.33 mm; TL 4.36-4.57 mm (stinger not included); PPL 0.22-0.24 mm; PPW 0.32-0.35 mm; PPH 0.34-0.36 mm; CI 113-116, SI 80-86, MaI 51-57, PI 83-87, PPI 141-151.

Worker description. Head. Head in full-face view slightly shorter than wide (CI 113-116) and maximum HW occurs behind the eyes, sides convex, occipital corners triangular, occipital margin strongly convex (Fig. 1). Thin lamella finely rugulose present on the anterolateral margin of the clypeus (Fig. 2). Eyes relatively large with EL approximately 1/5 of HL. Antennal scape long (SI 80-86); antenna with 12 segments, last three segments forming indistinct club. Clypeus short, anterior clypeal margin broadly convex with two angular projections on either sides. Mascitory margin 


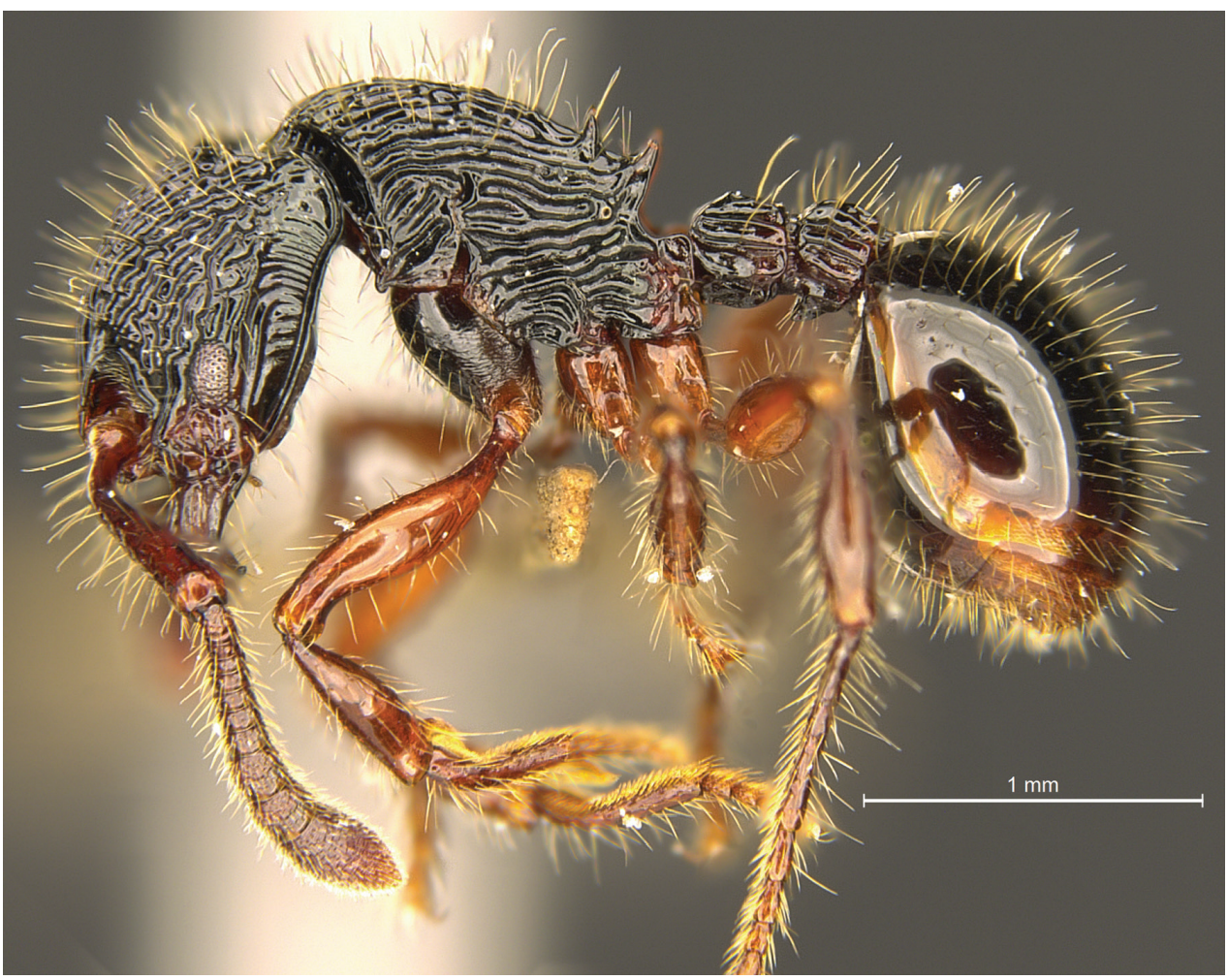

Figure 3. Profile view of Myrmecina magnificens (Holotype, ANTWEB1009004, LKCNHM) from Singapore.

of mandible with two apical teeth followed by a series of extremely reduced blunt denticles $(n=5)$.

Mesosoma. In profile view, dorsal outline of promesonotum flat to broadly convex, sloping along anterodorsal part of propodeum (Fig. 3). In profile view, propleuron angular and forming a distinct acute tooth. Two distinct propodeal spines both slightly longer than broad and pointing anteriorly (Fig. 4). Propodeal declivity strongly concave before terminating at a right angle with a rounded edge.

Metasoma. In profile view (Fig. 4), anterodorsal face of petiolar node broadly convex, posterodorsal face of petiolar node flat to weakly concave; overall dorsal outline of petiolar node triangular. Ventral outline of petiolar node rounded. Dorsal outline of postpetiole flat to weakly convex, sternopostpetiolar process well-developed and triangular, almost as long as broad and pointing anteriorly. Petiolar node is longer than postpetiole. In dorsal view (Fig. 5), petiolar node is broader than long and exhibits trapezoid shape; petiolar node is distinctly broader at posterior margin than at anterior margin. Postpetiole is broader than long and exhibits rectangular shape; width of postpetiole is similar at both the anterior and posterior margins. 


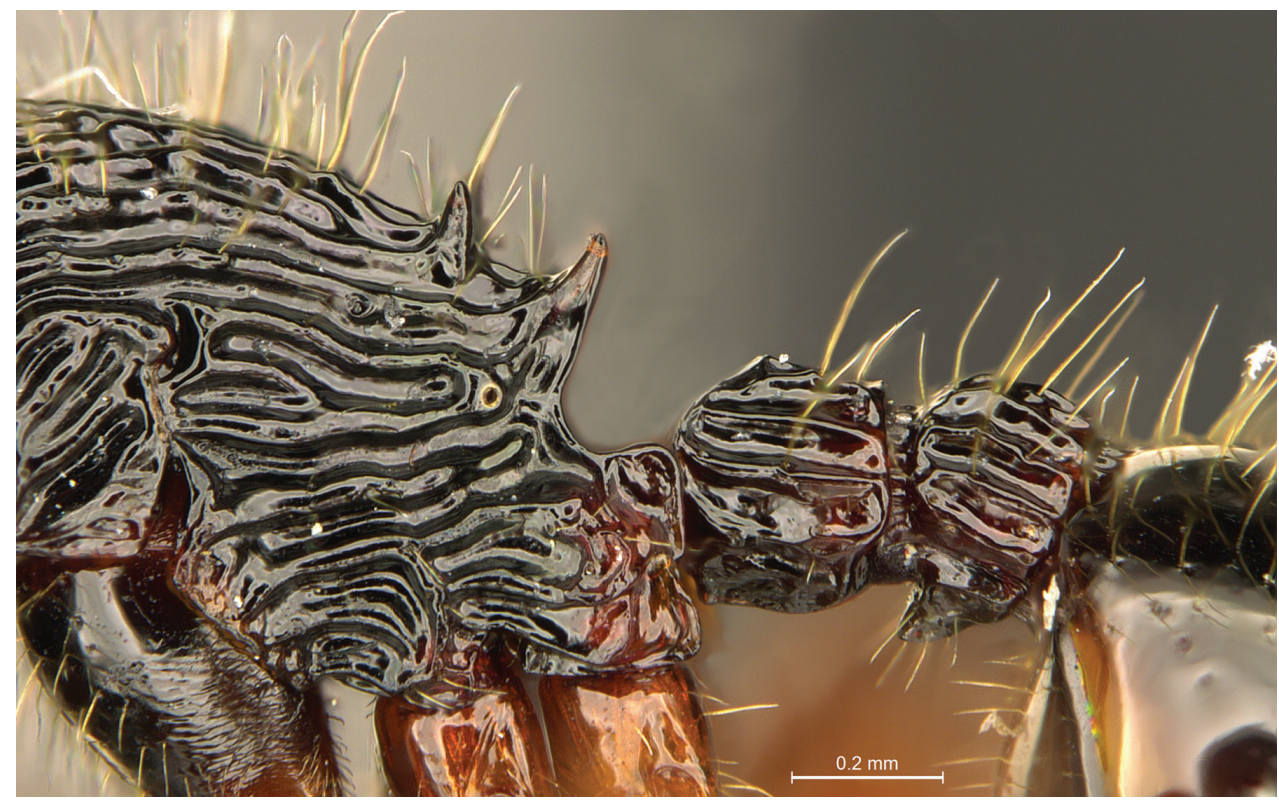

Figure 4. Profile view focusing on the propodeum, petiole and subpetiole of Myrmecina magnificens (Holotype, ANTWEB1009004, LKCNHM) from Singapore.

Sculpture. Sculpturing on head and thorax running longitudinally. Sculpture on head parallel on its middle portion and divergent on the sides and posterior portions. Groove on head with latitudinal sculptures present on most of its length. In profile view, sculpture on thorax presenting a complex pattern with parallel or and convergent sculptures (Fig. 3). Parallel sculptures present on the entire length of the petiole and postpetiole. Gaster smooth and shiny. In dorsal view, thorax entirely sculptured with a single median carina separating in two symmetrical portions the dorsal sculptures (Fig. 5). Other sculptures converging posteriorly to reach the median carina on the dorsum of the thorax.

Pubescence. Almost all of body covered in abundant erect long hairs $(0.2-0.25$ $\mathrm{mm}$ ) with the exception of the propodeal junction and ventral surface of the petiole where pubescence is absent.

Coloration. Most of head, mesosoma and gaster black; antenna, clypeus and mandibles dark red to dark brown; legs, posterior portion of propodeum light amber to light brown; pubescence and margins of gastral tergites yellow to light gold.

Castes. Male and gyne castes unknown.

Etymology. The species epithet is derived from the English word 'magnificent', referencing the beautiful appearance of this species, which makes it truly magnificent to behold. The species epithet is a noun, and thus invariant.

Distribution. Southeast Asia. Only known from Singapore.

Ecology. Almost nothing is known about the ecology of M. magnificens. All specimens were collected from a selectively logged lowland primary rainforest. Based on the 


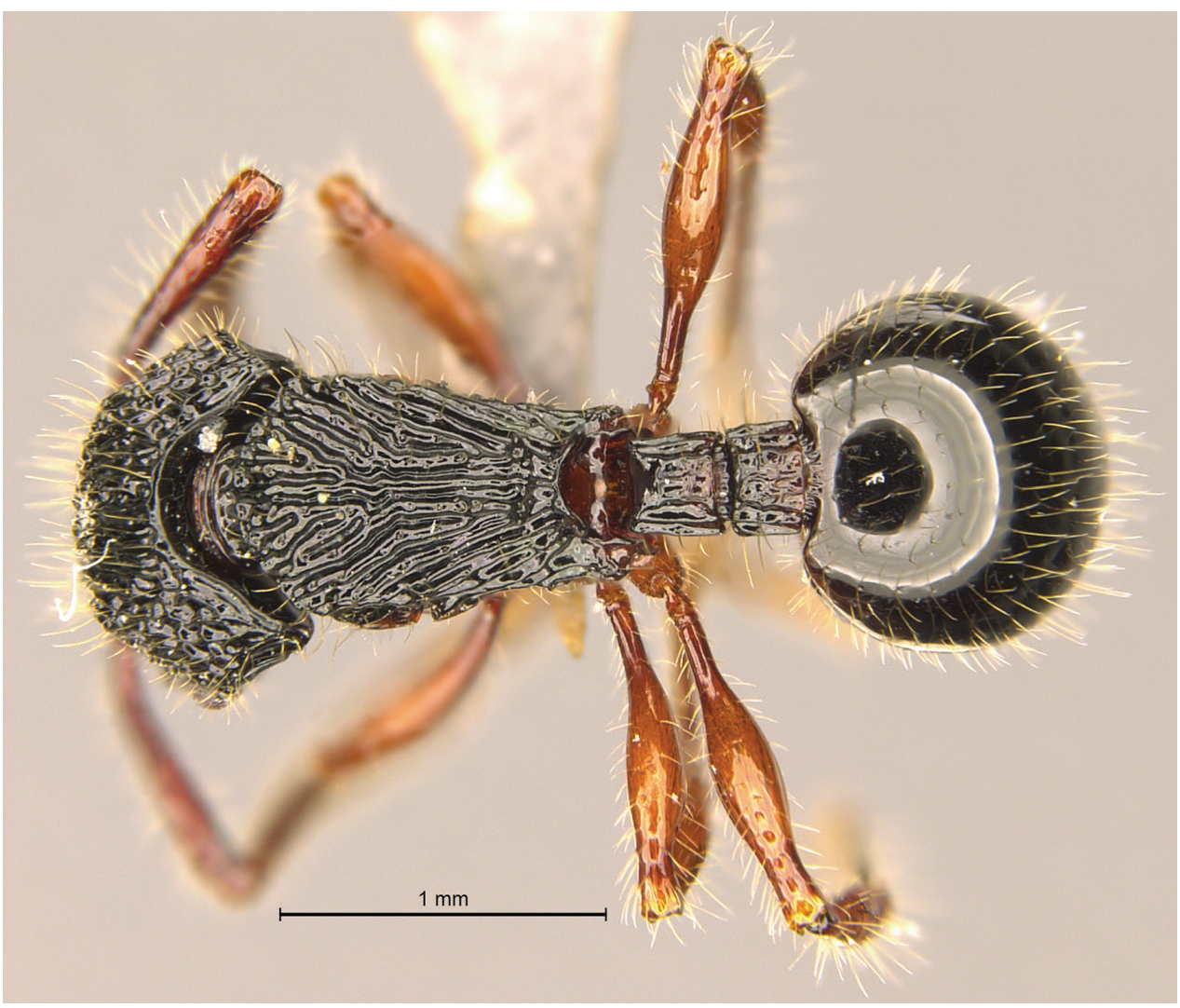

Figure 5. Dorsal view of Myrmecina magnificens (Holotype, ANTWEB1009004, LKCNHM) from Singapore.

collection methods used to collect several workers $(\mathrm{N}=5)$, we suspect that $M$. magnificens probably forages within the leaf litter and topsoil. This is based on our collection of the species via a Berlese extraction of sifted leaf litter and topsoil, as well as from a subterranean pitfall trap baited with tuna and buried at a depth of $5 \mathrm{~cm}$ underground. No other conspecifics were found in similar traps buried at greater depths (i.e. 15, 20, $25 \mathrm{~cm}$ ) within the same locality.

Diagnosis. With the exception of $M$. sulcata for which body size was not recorded in the species description, the new species $M$. magnificens can quickly be distinguished from other Myrmecina species in the Sundaland region by its large overall size (TL 4.2-4.6 $\mathrm{mm}$ ) and its distinctly long and forward-pointing propodeal spines. While $M$. nesaea, M. semipolita, M. sulcata and $M$. undulata also possess relatively long propodeal spines, their spines are posteriorly oriented. The other two species, $M$. bandarensis and $M$. butteli have relatively short propodeal spines, and are also notably smaller in their overall sizes (TL 1.9-2.2 mm), which by comparison would be approximately half that of M. magnificens. 


\title{
Synoptic species list of Myrmecina species known from Sundaland
}

\author{
Myrmecina bandarensis Forel, 1913 (Sumatra) \\ Myrmecina butteli Forel, 1913 (Sumatra) \\ Myrmecina magnificens sp. n. (Singapore) \\ Myrmecina nesaea Wheeler, 1924 (Sumatra) - described from gyne only \\ Myrmecina semipolita Forel, 1905 (Java) \\ Myrmecina sulcata Emery, 1887 (Java) \\ Myrmecina undulata Emery, 1900 (Borneo, Sumatra)
}

\section{Key to Myrmecina species from Sundaland based on worker caste}

A new key to Myrmecina species from Sundaland based on descriptions of the worker caste was developed from scrutinizing the respective taxonomic descriptions for the worker caste of M. butteli (Forel 1913, p.71), M. semipolita (Forel 1905, p.15), M. sulcata (Emery 1887, p.449) and M. undulata (Emery 1900, p.678). For two other Sunda Myrmecina species, specimens of the worker caste were examined. These included: M. bandarensis, one imaged specimen on Antweb.org (Syntype, Forel, 1913, Bandar Barae, Sumatra, CASENT0908953); M. magnificens sp. n., three mounted type specimens, all with same collection data, "M. Wong, 02.IX.2015, Seletar Trail, Singapore" (Holotype ANTWEB1009004, Paratypes ANTWEB1009005 to ANTWEB1009008). In an effort to be comprehensive, we also tentatively incorporated the description of $M$. nesaea, which was described from the gyne caste only (Wheeler 1924, p.247). For this last species, we only included characters on pilosity that might be shared between the gyne and worker castes into our comparative analyses. However a more detailed study of the type specimens of this species including a complementary description would represent an interesting contribution in the future.

1 In profile view propodeal spines pointing posteriorly

- $\quad$ In profile view propodeal spines pointing anteriorly; large sized species (TL > $4 \mathrm{~mm}$ ) (Singapore)

M. magnificens sp. $\mathbf{n}$.

2 In profile view propodeal spines small with length shorter than or equivalent to basal width 3 In profile view propodeal spines distinct with length exceeding basal width... 4 In profile view pro-mesonotum weak to moderately convex (Fig. 6b); in fullface view anterior edge of clypeus straight, eyes large, approximately $1 / 4$ of HL (Fig. 6a) (Sumatra)

M. bandarensis Forel

- In profile view pro-mesonotum strongly convex; in full-face view anterior edge of clypeus slightly concave (Sumatra)

M. butteli Forel

4 In full-face view clypeus with distinctly concave anterior edge (Borneo, Sumatra) M. undulata Emery

- $\quad$ In full-face view clypeus with straight anterior edge 


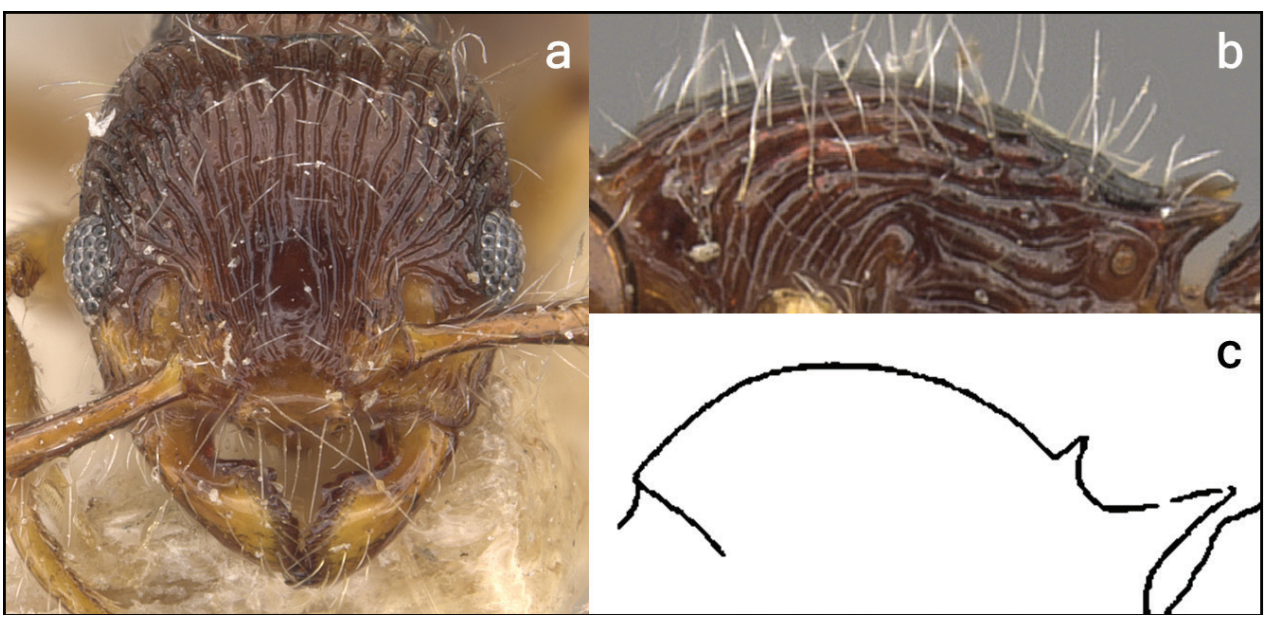

Figure 6. Photographs of M. bandarensis from AntWeb (CASENT0908953, photos by Will Ericson) in a full-face view showing large eyes and straight anterior edge of clypeus $\mathbf{b}$ profile view, close-up of thorax showing weak to moderately convex pro-mesonotum and short propodeal spine. Original illustration of $M$. sulcata in profile view $\mathbf{C}$ with close-up of thorax showing strongly convex pro-mesonotum and long propodeal spine pointing posteriorly at approximate forty-five degree angle (from Emery 1887, p.449, pl. 1, fig. 11)

5 In profile view pro-mesonotum strongly convex (Fig. 6c); propodeal spine with length that is almost twice of basal width, and pointing posteriorly at an approximate forty-five degree angle (Fig. 6c) (Java).......... M. sulcata Emery

- $\quad$ In profile view pro-mesonotum weakly convex; propodeal spines with length slightly exceeding basal width and pointing posteriorly at a nearly horizontal angle.

6 Pilosity on entire body long and white in colour (Sumatra)

M. nesaea Wheeler (Described from gyne only)

- $\quad$ Pilosity on entire body short and yellow in colour (Java)....M. semipolita Forel

\section{Discussion}

Myrmecina magnificens represents the first Myrmecina species described from the Malay Peninsula and completes the distribution of the genus in the Sundaland region. Although several records of Myrmecina have been reported from ecological work in this region, most of them have not been formerly identified. For example, many unidentified Myrmecina species were previously reported from Peninsular Malaysia: Negeri Selemban and Selangor provinces in Malaysia (Agosti et al. 1994; Malsch 2000), as well as Narathiwat, Songkhla and Trang provinces in Thailand (Noon-anant et al. 2005; Watanasit et al. 2007; Sakchoowong et al. 2015). In this regard, the key provided here is by no means exhaustive and it is likely that new Myrmecina species will be described in the future. Nevertheless, in light of the characteristic morphology of the new species 
described here, we took the opportunity to present a primary baseline for future work on this genus in the Sundaland region. Notably, while populations of M. magnificens have yet to be discovered in other parts of Singapore, the apparent abundance of this species within Seletar forest is surprising; thus far several specimens have been collected with a variety of methods including Berlese extractions of understorey material as well as baited subterranean pitfall traps. In conclusion, the present discovery of a new ant species that is non-cryptic, of relatively large body size ( $\mathrm{TL}>4 \mathrm{~mm}$ ) and perhaps not uncommon in a small forest remnant (ca. $2000 \mathrm{ha}$ ) of a highly urbanized city (i.e. Singapore) clearly highlights the shortage of extensive sampling in Southeast Asia, and is very encouraging to future myrmecological research in this part of the world.

\section{Acknowledgments}

The independent research work of the first author (MW) was partially supported by the generous contributions of numerous donors through experiment.com/antsofsingapore. The funders had no role in study design, data collection and analysis, decision to publish, or preparation of the manuscript. We are grateful to staff of the National Biodiversity Centre Singapore for their support with research permits. We would also like to thank Ying Luo and Lily $\mathrm{Ng}$ from the University of Hong Kong for their assistance with specimen handling and imaging. The authors would like to thank Brian Fisher and the team of Antweb.org for providing free access to the pictures. Finally, we thank Himender Bharti for his valuable comments on a previous version of the manuscript.

\section{References}

Agosti D, Mohamed M, Arthur CYC (1994) Has the diversity of tropical ant fauna been underestimated? An indication from leaf litter studies in a west Malaysian lowland rain forest. Tropical Biodiversity 2: 270-275.

Antmaps (2016) Antmaps. http://www.antmaps.org/ [accessed on February $2^{\text {nd }} 2016$ ]

Baroni Urbani C (1977) Materiali per una revisione della sottofamiglia Leptanillinae Emery (Hymenoptera: Formicidae). Entomologica Basiliensia 2: 427-488.

Bolton B (2016) AntWeb: Bolton World Catalog. http://www.antweb.org/ [accessed on February $2^{\text {nd }} 2016$ ]

Buschinger A, Schreiber M (2002) Queen polymorphism and queen-morph related facultative polygyny in the ant, Myrmecina graminicola (Hymenoptera, Formicidae). Insectes sociaux 49: 344-353. doi: 10.1007/PL00012658

Emery C (1887) Catalogo delle formiche esistenti nelle collezioni del Museo Civico di Genova. Parte terza. Formiche della regione Indo-Malese e dell'Australia (continuazione e fine). Annali del Museo Civico di Storia Naturale 25: 449-464.

Emery C (1900) Formiche raccolte da Elio Modigliani in Sumatra, Engano e Mentawei. Annali del Museo Civico di Storia Naturale 40: 661-722. doi: 10.5962/bhl.part.9035 
Fischer G, Azorsa F, Fisher B (2014) The ant genus Carebara Westwood (Hymenoptera, Formicidae): synonymisation of Pheidologeton Mayr under Carebara, establishment and revision of the C. polita species group. ZooKeys 438: 57-112. doi: 10.3897/ zookeys.438.7922

Forel A (1905) Ameisen aus Java. Gesammelt von Prof. Karl Kraepelin 1904. Mitteilungen aus dem Naturhistorischen Museum in Hamburg 22: 1-26.

Forel A (1913) Wissenschaftliche Ergebnisse einer Forschungsreise nach Ostindien ausgeführt im Auftrage der Kgl. Preuss. Akademie der Wissenschaften zu Berlin von H. v. ButtelReepen. II. Ameisen aus Sumatra, Java, Malacca und Ceylon. Gesammelt von Herrn Prof. Dr. v. Buttel-Reepen in den Jahren 1911-1912. Zoologische Jahrbücher. Abteilung für Systematik, Geographie und Biologie der Tiere 36: 1-148.

Huang J-H, Huang Y, Zhou S-Y (2008) A new species of the genus Myrmecina Curtis, 1829 (Hymenoptera, Formicidae) from Hunan Province, China. Acta Zootaxonomica Sinica 33: $275-278$.

Malsch A (2000) Investigation of the diversity of leaf-litter inhabiting ants in Pasoh, Malaysia. In: Agosti D, Majer J, Alonso L, Schultz T (Eds) Sampling Ground-Dwelling Ants: Case Studies from the World's Rain Forests. Curtin University School of Environmental Biology (Bulletin No. 18), Perth, 31-40.

Masuko K (1994) Specialized predation on oribatid mites by two species of the ant genus Myrmecina (Hymenoptera: Formicidae). Psyche 101: 159-173. doi: 10.1155/1994/96412

Noon-anant N, Watanasit S, Witawatwitaya D (2005) Species diversity and abundance of ants in lowland tropical rain forest of Bala forest, Narathiwat province, southern Peninsular Thailand. Natural History Bulletin of the Siam Society 53: 203-213.

Sakchoowong W, Hasin S, Pachey N, Amornsak W, Bunyavejchewin S, Kongnoo P, Basset Y (2015) Influence of leaf litter composition on ant assemblages in a lowland tropical rainforest in Thailand. Asian Myrmecology 7: 57-71.

Shattuck S (2009) A revision the Australian species of the ant genus Myrmecina (Hymenoptera: Formicidae). Zootaxa 2146: 1-21.

Steiner FM, Schlick-Steiner BC, Konrad H, Linksvayer TA, Quek SP, Chirstian E, Buschinger A (2006) Phylogeny and evolutionary history of queen polymorphic Myrmecina ants (Hymenoptera: Formicidae). European Journal of Entomology 103: 619-626. doi: 10.14411/ eje. 2006.083

Terayama M, Kubota S, Eguchi K (2014) Encyclopedia of Japanese ants. Asakura Shoten, Tokyo, 278 pp. [In Japanese]

Watanasit S, Saewai J, Philapplueng A (2007) Ants of the Klong U-Tapao Basin, Southern Thailand. Asian Myrmecology 1: 69-79.

Wheeler WM (1924) Ants of Krakatau and other islands in the Sunda Strait. Treubia 5: 239-258. Zhou S, Huang J, Ma L (2008) Two new species of the ant genus Myrmecina (Hymenoptera: Formicidae), with a key to Chinese species. Sociobiology 52: 283-291. 